\title{
¿Enseñanza de las ciencias por disciplinas $o$ interdisciplinariedad en la escuela?
}

Resumen: El artículo presenta algunos supuestos que están a la base de la incorporación de los saberes en el currículo de la escuela y cómo ello responde a una manera de entender la relación con el conocimiento desde la que se naturaliza la fragmentación de los saberes. Describe algunos argumentos que abogan por una relación interdisciplinaria con los saberes que hace explícita la necesidad de transformar las relaciones con el conocimiento que se propician en la escuela. Además, discute algunas propuestas que constituyen una orientación de la forma en que diferentes grupos y comunidades académicas han construido una respuesta para proveer a los ciudadanos de una relación más cercana con los productos de la ciencia y la tecnología.

Palabras clave: Interdisciplinariedad, disciplinas científicas, enseñanza de las ciencias, curricularización de los saberes.

\author{
Steiner Valencia Vargas \\ Olga Méndez Núñez \\ Gladys Jiménez Gómez*
}

Artículo recibido: 15-5-2007 y aprobado: 30-4-2008

¿Science teaching by disciplines or interdisciplinarity at the school?

Abstract: This paper presents some considerations about the ways in which knowledge is included in the school curricula. The process is an answer to the need of understanding the relationships between knowledge and disciplines in the school contexts, when the first is broken down. The theory describes some arguments in favour of an interdisciplinary relation which expresses fully and clearly the necessity of changing the relationships that are implemented in the school regarding to knowledge. Some proposals of the academic community are discussed and different strategies are designed by groups of teachers in order to illustrate how it is possible to promote a meaningful relationship between the citizens and the products of science and technology.

Key Words: Interdisciplinary knowledge, scientific disciplines, Sciences teaching, curriculum. 


\section{Presentación}

Los establecimientos educacionales actuales se comportan aún como si el mundo pudiera dividirse tajantemente en las categorías de la ciencia del siglo XIX: La realidad física, el mundo viviente, y la esfera de la intencionalidad y la acción humanas. Ervin Laszlo

El desbordamiento del mercado tecnológico, la invasión de las nuevas tecnologías de la información en lo más cotidiano de nuestras vidas y el privilegio cultural hacia los conocimientos productos de la ciencia y la tecnología imponen al ser humano contemporáneo nuevas maneras de ser y estar que hacen inexorable su condición de consumidor. Sin embargo, frente a esta condición puede optarse por una actitud pasiva o crítica ante la posibilidad de transformar las relaciones con el entorno y con las dinámicas de producción de saber y con los productos tecnocientíficos.

Esta opción se relaciona directamente con el papel que la escuela desempeña frente a las relaciones que promueve con el conocimiento, pues la condición de alfabetización en cuanto a manejo de los códigos que permiten leer y escribir quedó trascendida; ahora el imperativo es otro, es importante participar de otro tipo de alfabetización que permita asumir creativa y críticamente un papel frente a las dinámicas de saber que se despliegan en el mundo contemporáneo. Desde esta perspectiva, se hace prioritario que la escuela provea de herramientas que permitan a los sujetos relacionarse críticamente con la ciencia y la tecnología, pues de lo contrario quedaremos reducidos a la condición de espectadores pasmados ante la prolífica variedad de productos tecnocientíficos, donde el sentido de lo que se hace en el aula de clase pierde vínculo con el mundo que habitamos y frente al cual tenemos que tomar decisiones concretas.

Así puede afirmarse que en este nuevo paisaje social y cultural el papel de la escuela como difusora de información se ha agotado. Es pues en una nueva perspectiva como nos hallamos ante unas necesidades y exigencias sociales, que si bien la escuela no puede resolver en su totalidad, sí puede apoyar en la medida en que construya un sentido para las prácticas que allí se desarrollan y asuma un papel crítico frente a la sociedad tecnocientífica con la cual hemos de relacionarnos. Reconocemos que la escuela no puede ser sustituida, que si bien ella y los maestros han sido sustituidos en su labor de informadores, no lo han sido en su participación en la construcción de espacios de socialización que fortalecen la autonomía y la subjetividad, redimensionan las relaciones con el conocimiento y la cultura, y propenden por construir sociedades más democráticas que se piensen y proyecten colectivamente (Arellano, 2005).

Desde el reconocimiento de estos retos y exigencias que se le plantean a la escuela, se considera importante desarrollar análisis que permitan definir el tipo de educación científica que deseamos ofrecer a nuestros estudiantes para abordar comprensivamente una relación con el entorno natural y social. Estos análisis cruzan por plantearnos interrogantes acerca de: ¿Qué tipo de enseñanza es solidaria con una actitud crítica frente a los productos tecnocientíficos? ¿Cuál es el papel del maestro frente a los desafíos que le plantea un mundo tecnológica y científicamente 
prolífico? ¿Cuál es el papel de los saberes disciplinares en la escuela?

Preguntas como estas animan las elaboraciones que planteamos en este artículo, para lo cual en un primer apartado se identifican algunos de los supuestos que están a la base de la curricularización de los saberes en la escuela y cómo ello responde a una manera de entender la relación con el conocimiento desde la que se ha naturalizado la fragmentación de los saberes. En un segundo apartado, describimos algunos argumentos de tipo cognitivo, epistemológico y social que abogan por una manera de relación interdisciplinaria con los saberes haciendo explícita la necesidad de transformar las relaciones con el conocimiento que se propician en la escuela.

Este último interés ha movilizado búsquedas que tienen que ver con la definición de metas y la construcción de didácticas más adecuadas para tal logro. Entre estas búsquedas, la propuesta Ciencia, Tecnología y Sociedad, CTS, y Alfabetización Científica y Tecnológica, $\mathrm{ACT}$, constituyen una orientación clara de la forma en que diferentes grupos académicos y sociedades han construido una respuesta para proveer a los ciudadanos una relación más cercana con los productos de la ciencia y la tecnología.

\section{Las disciplinas: Curricularización del conocimiento científico en la escuela}

Cuando nos asomamos a entender el mundo físico, biológico, cultural en el que nos encontramos, es a nosotros mismos a quienes descubrimos y es con nosotros mismos con quienes contamos.

Edgar Morin

Estudios en historia y epistemología de la ciencia muestran que uno de los logros culturales más significativos del siglo XIX lo constituye la consolidación de unos corpus teórico-experimentales denominados disciplinas científicas (Kuhn, 1989; Lakatos, 1983; Jacob, 1988; Bachelard, 1976). Dichas disciplinas (biología, física y química) han tenido un gran impacto en la definición y desarrollo de prácticas socioculturales como la política, la agricultura, la economía, la salud y la industria en general.

El éxito de la influencia de estas disciplinas en las dinámicas culturales ha estado, de alguna manera, garantizado por las formas de relación con estos saberes constituidos que se promueven en la escuela. Es reconocido el peso que desde la escuela básica se da a la formación en los contenidos disciplinares como garantía para acceder y lograr un buen desempeño en la formación universitaria. Desde la formación inicial el estudiante es instalado en un complejo de teorías y de conceptos plenamente diferenciables, que objetivan los productos de la ciencia y la hacen aparecer como opción única y verdadera de relación con el mundo.

En este sentido, desde la escuela básica hasta la formación profesional, las prácticas educativas promueven unas relaciones de identidad entre los contenidos a enseñar y los conocimientos disciplinares. Estas relaciones identitarias, generalmente, se expresan en la transposición de métodos, procedimientos y conceptos, en la cual se desconocen tanto los procesos históricos de constitución de las disciplinas como los criterios desde los cuales se asumen como espacios diferenciados de conocimiento. Es decir, que tales prácticas, por omisión o por ignorancia, no consideran relevante mostrar que las disciplinas son 
el resultado de ordenar una multiplicidad de prácticas y saberes y organizar los hallazgos teórico-experimentales de una época.

Aquí se hace explícita la analogía que no siempre es sujeta a revisión por los maestros y es la que se plantea entre las disciplinas científicas y las disciplinas escolares (Fourez, 1994). Pues si bien es cierto que en la escuela se enseña física, química y biología, entre otras, no siempre estos cuerpos conceptuales, las metodologías que se definen o que se promueven en su enseñanza se corresponden con los cuerpos disciplinares que tienen una constitución histórica y que responden a unas necesidades explicativas socioculturalmente determinadas, en las cuales se han configurado unos objetos de conocimiento que las hacen definibles y sustentables epistemológicamente.

Desde esta perspectiva, lo que se inauguró como una organización de los saberes y que posibilitó proyectar sus desarrollos en un momento histórico determinado, se ha traducido en la institucionalización de las disciplinas y en su consiguiente estructuración en currículos cada vez más segmentados que coartan las posibilidades creativas para explicar el mundo y las condicionan a la asunción de teorías, principios, normas, reglas y métodos.

Habría que ver, entonces, si los procesos de curricularización de los saberes en la escuela incluyen las características que definen a las disciplinas y si este proceder vincula la claridad en torno a su objeto de estudio, prácticas metodológicas, tradiciones fundantes y léxico propio, pero, ante todo, en cómo estas características definen una manera de relacionarse con el mundo que permite, como lo plantea Guidoni (1990), constituirse en una proyección de la realidad que hace ver el mundo bajo una óptica específica.

Sin embargo, lo que se termina promoviendo en la apropiación que se hace de ellas en la escuela está lejos de brindar unas herramientas conceptuales y metodológicas que permitan a los estudiantes comprender el mundo desde la óptica de la física, la química o la biología, pues su estudio se restringe a la apropiación de contenidos considerados estructurantes en cada campo disciplinar sin que se tenga acercamiento ni comprensión de a qué problemas responden, qué explican o por qué se hacen necesarios en las formas de relacionarnos con el mundo natural y social.

Así, ante la ausencia de un sentido para abordar las disciplinas en la escuela, se formulan didácticas desde las cuales se adecuan, seleccionan y ponen en condiciones de enseñanza lo que ya se considera que define los campos disciplinares estatuidos (conceptos, leyes, principios, metodologías, etc.), sin que en ellas medie la forma en que se resuelven problemas del entorno. Tal motivación obedece, por una parte, a que usualmente se asume que la didáctica dota a las personas de los contenidos conceptuales, actitudinales y procedimentales propios de las ciencias desde los cuales pueden enfrentar tanto cuestiones científico-técnicas, como situaciones de la vida cotidiana y, por otra, porque se considera que las disciplinas permiten pasar de las representaciones espontáneas a las científicas, consideradas estas últimas como pertinentes y deseables para habitar el mundo contemporáneo. 
Además, las disciplinas constituyen un dominio de la cultura en el que algunos consideran necesario instalarse como ciudadanos, ya que les permite hacerse partícipes de los desarrollos culturales de la humanidad y apropiar unos saberes y valores que se consideran importantes en sí mismos por el carácter de legitimidad que social e históricamente han adquirido, sin importar si representan alteraciones de las formas mismas de relacionarse con el mundo natural y social.

Desde estas consideraciones, podemos afirmar que la enseñanza de las ciencias se erige como un proyecto cultural, que en el caso colombiano busca

...ofrecerle a los estudiantes la po-

sibilidad de conocer los procesos

físicos, químicos y biológicos y su

relación con los procesos culturales, en especial aquellos que tienen la capacidad de afectar el carácter armónico del ambiente. Este conocimiento debe darse en el estudiante en forma tal que pueda entender los procesos evolutivos que hicieron posible que hoy existamos como especie cultural y de apropiarse de ese acervo de conocimientos que le permiten ejercer un control sobre su entorno (Lineamientos Curriculares. MEN. 1998. La cursiva es nuestra).

Es claro que en argumentos como estos lo que se expresa es una confianza en que la adquisición de los conocimientos científicos proveen las condiciones para llevar una vida más adecuada, comprensiva y armónica de relacionarnos con los otros y con el entorno natural y social. Más que involucrar a los estudiantes en la actividad misma de la producción de conocimiento, se les instala en una relación de exterioridad con unos productos elaborados por unas mentes ex- cepcionales, decisión que opera desde el supuesto que "el conocimiento científico y el tecnológico son productos sociales en tanto que el conocimiento común es más un acontecimiento individual" (Lineamientos Curriculares, MEN, 1998). Desde esta perspectiva, se hace énfasis en las ciencias exactas, específicamente en la matemática, la física, la química y la biología, cuyas lógicas de organización y producción de conocimiento se consideran imperantes y extrapolables a las formas de organizar la enseñanza de las ciencias en la escuela.

Ahora bien, dada la naturalidad con que se asume la enseñanza de las ciencias por disciplinas en la escuela, se hace necesario interrogar por el origen de ellas y por el carácter de objetividad con que se han asumido hasta llegar a considerarlas como "entidades" dadas que no precisan el estudio de las condiciones de su aparición. Tal análisis permite considerar que los saberes disciplinares se estatuyen en virtud de intereses, definición de problemáticas, desarrollo de proyectos y compromisos de las comunidades científicas en una época dada, y que son estas las condiciones que dan origen a campos de estudio particulares (Latour, 1989).

En este sentido, frente a la imagen que considera las disciplinas como estructuras que conjugan teorías sancionadas, leyes universales y explicaciones únicas y verdaderas, oponemos la imagen según la cual el surgimiento de los corpus teórico-experimentales que sustentan los saberes disciplinares se comprende desde sus dinámicas de estructuración, los contextos de explicación donde se originan, la solución que dan a las problemáticas que se plantean y los procesos de formalización que subyacen 
a sus teorías. A la comprensión de estas dinámicas le es solidaria una concepción de ciencia como actividad de la cultura (Ayala, 2005), desde la cual se asigna un carácter social e histórico al conocimiento científico y se asume la consolidación de las disciplinas como un proceso histórico en el que se validan y legitiman procederes experimentales y teóricos frente a problemáticas específicas.

Análisis como los planteados permiten trascender la comprensión de las disciplinas desde la institucionalización que promueven respecto a un saber y desde el tipo de relaciones que en diferentes ámbitos de la cultura se establecen con los productos de su especialización y que han llevado a asumirlas desde una visión estática y cerrada.

\section{Interdisciplinariedad: ¿una manera para conocer el mundo?}

Los distintos saberes se contemplan como iluminaciones locales que a pesar de todo es importante poner en comunicación; ahí es donde las prácticas interdisciplinares adquieren toda su importancia.

Gerard Fourez

El cuestionamiento a la enseñanza por disciplinas ha planteado la necesidad de transitar hacia propuestas que respaldan el desarrollo y promoción de formas de trabajo interdisciplinario en la escuela y en diferentes ámbitos de la vida moderna. Así aparecen diferentes formas de legitimar la pertinencia de la interdisciplinariedad que recurren a argumentos que podemos agrupar en tres tipos: cognitivos, epistemológicos y sociales; estos argumentos no se presentan de manera aislada en los discursos, sino que incluso se mezclan y hacen más fuerte la persistencia de las miradas interdisciplinarias para abordar cierto tipo de prácticas.

El desarrollo de la psicología cognitiva, de la epistemología genética y de lo que actualmente se denominan ciencias cognitivas, ha aportado criterios desde los cuales se da cuenta de los procesos, mecanismos e instancias involucradas en el aprendizaje. Este tipo de desarrollos ha planteado retos a la escuela y a la enseñanza de las ciencias, en la medida en que muestra que los sujetos organizan fenómenos y vivencian el mundo de una forma holística y compleja; es decir, como un todo organizado.

En los enfoques que orientan diversas prácticas de enseñanza de las ciencias se han traducido estos planteamientos como argumentos cognitivos que cuestionan la segmentarización de los saberes y conducen a comprender la dinámica global en la que el sujeto se aproxima a los fenómenos como una forma interdisciplinaria de relación con los saberes; esta forma interdisciplinaria, sin embargo, se hace cada vez más definible y delimitable de acuerdo con el enriquecimiento de la experiencia del sujeto y la capacidad de abordar problemas más específicos. Así, autores como Guidoni (1990) consideran que "no tiene probablemente ningún sentido proponer a los niños modelos especializados y rigurosos como guía para la interpretación de su experiencia". No obstante las posibilidades de tener relación con esos saberes disciplinares se acrecientan en la medida en que el sujeto accede a un nuevo campo de experiencias y puede concebir las disciplinas como diferentes proyecciones de la realidad o complejos 
de instrumentos (abstractos) especializados para conocer. Sin embargo, se mantiene el argumento de que

... sobre todo en un contexto escolar, es particularmente importante desarrollar un modo de conocer no fragmentario, aún cuando necesariamente sectorizado en los distintos criterios con que deben aprenderse las diversas disciplinas (o asignaturas)... que al mismo tiempo no nieguen y no estén en contraste con las evidencias del vivir y del conocer cotidianos (Guidoni, 1990).

Otros argumentos son de tipo epistemológico y recurren a la restricción comprensiva que imponen las disciplinas para abordar la complejidad del mundo. Una de las posturas que podemos referenciar en esta línea tiene asidero en los discursos instalados en las nuevas teorías de la ciencia (teoría del caos, teoría de la complejidad) que plantean la incompletitud del saber y asumen el riesgo que sustenta la complejidad de los fenómenos como posibilidad de ingresar a un mundo más rico y creativo. Desde esta perspectiva, la interdisciplinariedad emerge como posibilidad de realizar acciones más integrales y como una postura crítica frente a las disciplinas, que renuncia a una pretensión totalizante. A algunas posturas insertas en este tipo de argumentos se les critica, sin embargo, la forma en que asumen que la interdisciplinariedad es la promesa para llegar a un saber completo que puede abarcarlo todo para cubrir con el manto de los distintos saberes aquello que aparece inabarcable.

Discursos como los de Edgar Morin se constituyen en una base que ejemplifica este tipo de posturas y que abogan por la necesidad de una transformación multidimensional de aquello que entendemos por ciencia, que compete por ejemplo, a transformar unos de sus imperativos, la inevitabilidad de la parcelación disciplinaria y el fraccionamiento teórico. Con ello se asiste a la necesidad de construir y concebir una nueva ciencia que lo involucre todo, pero sin borrar la individualidad de las disciplinas; aparentemente podría tratarse de una visión abarcante y avara de la ciencia, pero se trata más de una concepción que asuma la indecibilidad como constitutiva de sí misma. En términos del mismo Morin (1998), se trata de "desarrollar un pensamiento lo menos mutilante posible y lo más racional posible". Con ello se supera la barrera aparentemente infranqueable del lenguaje disciplinario que hace incluso sentir neófito a cualquier especialista frente a otro campo disciplinar específico.

La interdisciplinariedad que propone Morin no implica inter de relación que unifica pero que no diferencia; se trata de asumir una postura donde sea posible la unidad que pretende establecer relaciones enriquecedoras, pero respeta la identidad de las disciplinas, sin tratar con ello de afirmar que las disciplinas en sí mismas tienen una identidad, pues el asunto está en poner en cuestión la misma idea de identidad, para ello es necesario acudir a la historia de las ciencias que muestra cómo las fronteras nunca han sido claras y diferenciables, se trata más bien de una tensión permanente, en la que se construye a partir de la relación y el contraste.

Un tercer tipo de argumentos se plantea desde estudios de carácter sociológico. Estos argumentos se insertan 
en el pragmatismo, que muestra la complejidad de los problemas planteados y la necesidad de empezar a resolverlos desde visiones más integradoras. Desde estos argumentos adquieren sentido algunas investigaciones desarrolladas por profesionales que desde diferentes áreas del conocimiento exponen miradas especializadas sobre un objeto de estudio y que al conjugarse y yuxtaponerse garantizan la complejidad del objeto en su totalidad. De la misma manera, el desarrollo de la actividad científica en la configuración de problemáticas que inicialmente se presentaban como disciplinares ha mostrado la pertinencia de aunar esfuerzos teóricos y experimentales de los diferentes saberes conduciendo a la profusión de proyectos que se consideran interdisciplinarios, como el proyecto Manhattan, los proyectos Genoma o los proyectos Biosfera.

Este tipo de proyectos se configuran como escenarios téorico-experimentales desde los cuales se esgrimen argumentos que solicitan superar las brechas disciplinares para abordar la complejidad cultural a la que nos enfrentamos; es decir, los últimos desarrollos científicos y tecnológicos y su incidencia en prácticas sociales como la enseñanza, la economía, la política, la industria, entre otras. De hecho encontramos discursos sobre la reconfiguración de la ciencia contemporánea a la que se le plantea una revolución interdisciplinaria. $\mathrm{Al}$ respecto Laszlo afirma que:

La actual revolución de la ciencia es diferente de las anteriores porque es verdaderamente interdisciplinaria. Y se produce porque no hay razones, científicas o sociales, para seguir adhiriendo a teorías parchadas dentro de disciplinas únicas. Los científicos podrían producir una teoría general, matemáticamente rigurosa y al mismo tiempo abarcadora, de un universo en desenvolvimiento, en la cual la vida, la sociedad, el homo y la civilización humanas no sean ya elementos extraños entre sí o subproductos accidentales, sino partes integrantes del cambio y desarrollo (Laszlo, 1997).

Esta visión interdisciplinaria resulta ilustrativa para mostrar cómo la comunidad científica quiere hacerle frente al carácter reduccionista y discriminador de las disciplinas y a sus objetos de estudio definidos como originarios e inamovibles.

Por último, resulta interesante ver cómo frente a la retórica de la interdisciplinariedad que se instaura con fuerza en los contextos académicos se abren espacios alternos que plantean un discurso crítico frente a las posturas totalizantes que ya han mostrado su ineficacia y limitaciones para resolver problemas contextuales de comunidades específicas (Escobar, 2005). En este sentido, se promueve una conciencia vigilante frente a las posturas totalizantes que como el paradigma del pensamiento único irrumpen en diferentes ámbitos de la cultura y permean las prácticas sociales en la perspectiva de establecer procederes homogéneos y globalizantes que no consideran los contextos específicos de la acción. Dicha conciencia debe actuar con sigilo frente a discursos que como el de la interdisciplinariedad parecen adquirir una legitimidad per se y bajo los cuales pueden coexistir posturas que no siempre son solidarias con el carácter cultural y dinámico del conocimiento científico. 


\section{Perspectivas interdisciplinarias para la enseñanza de las ciencias}

Sí efectivamente, el espíritu humano no puede aprehender el enorme conjunto del saber disciplinario, hace falta, entonces, cambiar, ya sea al espíritu humano, ya sea al saber disciplinarizado.

Edgar Morin

Para todos es común afirmar que la enseñanza de las disciplinas en la escuela se ha instituido con tal obviedad que casi nunca se cuestiona su pertinencia, es tal el carácter de verdad con el que se erige, que ha logrado adquirir "vida" propia y las "asignaturas" en que se implementa han dejado de ser espacios conceptuales en diálogo en la escuela. El interés por reflexionar alrededor de tal obviedad ha llevado a maestros, grupos académicos y sociedad en general a hacerse partícipes de la discusión sobre la interdisciplinariedad y las generosidades de tal perspectiva para la enseñanza de las ciencias, esto en la medida en que en ella se anuncia la promesa de adquirir el tipo de procederes requeridos para actuar en un mundo donde los saberes especializados cada vez más necesitan ponerse en diálogo.

Un trabajo de exploración de la interdisciplinariedad como perspectiva para la enseñanza de las ciencias amerita dar cuenta de propuestas como las que están a la base del movimiento CTS (Ciencia, Tecnología y Sociedad) y ACT (Alfabetización Científica y Tecnológica), pues ellas recogen la orientación de una educación interdisciplinaria como una manera de abordar en la enseñanza problemas como los que habitualmente enfrentan los estudiantes y que afectan su cotidianidad, garantizando con ello una adecuada formación para la toma de decisiones informadas y acciones responsables (Membiela, 2001).

Propuestas como la ACT y la CTS parten de la idea de que para estudiar una determinada cuestión de la vida cotidiana, se precisan múltiples aproximaciones que llevan a vincular los saberes especializados y disciplinarios con los saberes cotidianos y la cultura común, con el fin de obtener soluciones efectivas para generar actuaciones autónomas y pensamiento crítico. Argumentos de este tipo hacen que propuestas como estas ganen un inusitado entusiasmo en maestros y grupos sociales que ven en ellas la solución a la necesaria

...alfabetización en ciencia y tecno-

logía, de manera que se capacite a

los ciudadanos para participar en

el proceso democrático de tomas de decisiones y se promueva la acción ciudadana encaminada a la resolución de problemas relacionados con la ciencia y la tecnología en nuestra sociedad (Membiela, 2001).

La razón que moviliza esta gran acogida es que en ellas se hace eco a una preocupación cada vez más sentida por maestros, la sociedad en general y, por supuesto, los gobiernos que definen las políticas educativas; y es la necesidad de establecer vínculos entre la escuela, los saberes científicos y tecnológicos y la configuración de sociedades cada vez más preparadas para los mercados laborales y alfabetizadas en las formas de abordar un mundo científico-tecnológico cada vez más complejo.

No obstante, ante tanta magnificencia que se expresa en propuestas como estas habría que hacer beneficio de inventario acerca de cuál es el imaginario que se promueve claramente acerca de la ciencia y la tecnología y cuáles son 
las formas de relación con los saberes que intentan instituir en la escuela. Es claro que dichas propuestas no ponen en cuestión la misma legitimidad del conocimiento científico y tecnológico, más bien intentan ponerlo en diálogo con la cultura de base de manera que se inserte paulatinamente y haga parte de las formas cotidianas de proceder de los ciudadanos. A la base de esto está un interés por inscribir la condición del consumidor culto y responsable en las sociedades sin poner en cuestión el problema de las dinámicas de producción de los saberes y mucho menos sin preocuparse por hacer partícipe al grueso de la población de la actividad de construcción de explicaciones del mundo natural y social que anima la producción de saber científico-tecnológico.

Otro aspecto sobre el que habría que reflexionar es la manera en que desde estas propuestas se asume una posición crítica con las prácticas de enseñanza, pero no con la manera en que se legitima y hace hegemónico el pensamiento científico, esto es, con las dinámicas de difusión del conocimiento. Queda pues planteado que la ciencia es un bien en sí mismo, del que debe apropiarse el ciudadano, la criticidad se remite así, a una cuestión de carácter metodológico y didáctico más que ideológico.

Consideraciones como las anteriores muestran que la decisión de orientar prácticas de enseñanza de las ciencias desde las disciplinas u optar por la interdisciplinariedad plantea al maestro la necesidad de tomar postura frente a su hacer y a los privilegios que establece para comprender cómo sus estudiantes se relacionan con los saberes, bien sea para inscribir su acción en la de difusor de la ciencia y la tecnología o en la de promotor de dinámicas de producción de conocimiento en la escuela. Si bien dicha decisión plantea al maestro la exigencia de hacer una revisión crítica de la noción de disciplina para superar su obviedad, también le plantea la necesidad de cuestionar los intereses que movilizan las opciones interdisciplinarias para la escuela y las relaciones de saber-poder que desde ellas se promueven. Asumir la interdisciplinariedad como una posición crítica frente a la enseñanza por disciplinas que renuncia a una pretensión totalizante y que pone de presente la incompletitud de los saberes, también plantea al maestro la exigencia de asumir los compromisos disciplinares de rigor y ejercicio crítico que su práctica le plantea, así como los compromisos culturales que la sociedad le demanda.

Desde la anterior perspectiva, propuestas como la CTS y la ACT resultan interesantes en la medida en que configuran un campo que solidariza las relaciones disciplinares y propone alternativas para la enseñanza de las ciencias, donde estudiantes y maestros vinculan diferentes ámbitos de conocimiento, intereses, sentires y formas de relacionarse con el mundo de manera holística y no segmentada. Sin embargo, reconocemos que abordar tal perspectiva amerita del maestro una conciencia vigilante frente a los riesgos de superficialidad, pragmatismo y confusión que pueden plantearse cuando se quiere responder a prácticas de enseñanza más contextuales. $\boldsymbol{\Delta}$ 


\section{Referencias}

Ayala, M. M. (2006, Jan/abr.). Los análisis histórico-críticos y la recontextualización de saberes científicos. Construyendo un nuevo espacio de posibilidades. Revista Pro-posiçoes, 17,1 (4), pp.19-38.

Bachelard, G. (1976). El materialismo racional. Buenos Aires: Paidós.

Escobar, A. (2005). Más allá del Tercer Mundo, globalización y diferencia. Bogotá: Instituto Colombiano de Antropología e Historia.

Fourez, G. (1994). La construcción del conocimiento científico. Madrid: Narcea.

Guidoni, P, Arca, M. y Mazzoli, P. (1990). Enseñar ciencia. Madrid: Paidós Educador.
Kuhn, T. S. (1962). La estructura de las revoluciones científicas. México: Fondo de Cultura Económica.

Lakatos, I. (1983). La metodología de los programas de investigación científica. Madrid: Alianza.

Latour, B. (1989). Joliot: punto de encuentro de la historia y de la física. En: Serres, M. Historia de las ciencias. Madrid: Cátedra.

Lazslo, E. (1997). La gran bifurcación. Barcelona: Gedisa.

Membiela, P. (2001). Una revisión del movimiento CTS en la enseñanza de las ciencias. En: Enseñanza de las ciencias desde la perspectiva CTS. Madrid: Narcea. 